\title{
Proton and Deuterium NMR study of the CBC9CB Dimer System
}

\author{
J. Carvalho, ${ }^{\dagger}$ C. Cruz ${ }^{*}, \dagger$ J. L. Figueirinhas ${ }^{*},{ }^{\dagger}$ M. G. Tamba,${ }^{\ddagger}$ A. Kohlmeier,${ }^{\ddagger}$ and \\ G. H. Mehl ${ }^{\ddagger}$ \\ $\dagger$ Department of Physics, and CeFEMA, Instituto Superior Técnico, Universidade de \\ Lisboa, Av. Rovisco Pais 1, 1049-001 Lisboa, Portugal \\ $\ddagger$ Department of Chemistry - University of Hull, Hull, HU6 7RX, United Kingdom
}




\begin{abstract}
Using proton and deuterium NMR spectroscopy, this work provides a description of the molecular orientational order of the mesogenic groups in both the $\mathrm{N}_{t b}$ and the classical nematic phase $(\mathrm{N})$, of the liquid crystal dimer CB-C9-CB. The proton NMR data were collected at high field (7 Tesla) with the nematic domains aligned by the field. Deuterium NMR data obtained from aligned samples at 11.7 Tesla, published by Hoffmann A. et al. Soft Matter 2015, 11, 850 were also considered in this study. Using first order perturbation theory, we carry out detailed simulations of the proton spectra from the terminal mesogenic groups along with the quadrupolar splittings from the $\mathrm{CD}$ bonds in the first chain positions, which allow for the determination of the Saupe order tensor for the mesogenic groups. We show that the main mechanism that induces the change of the ${ }^{1} \mathrm{H}$ NMR spectrum and the quadrupolar splittings at the $\mathrm{N}-\mathrm{N}_{t b}$ phase transition is the change in the orientation of the most ordered molecular frame (eigenframe of the Saupe tensor), along with the onset of the molecular biaxility parameter, D. This orientation change is associated to the achiral symmetry breaking at the $\mathrm{N}-\mathrm{N}_{t b}$ phase transition.
\end{abstract}

\title{
Introduction
}

Up to 2010 only four distinct nematic ground states had been found, ${ }^{1}$ for achiral molecular systems, the uniaxial nematic and the biaxial nematic; for chiral molecules the helical nematic and the blue phases. In 1973, however, Meyer suggested the theoretical possibility that molecular interactions that favour bend deformations might lead to a twist-bend nematic phase, in which the director will have an helical distribution with a constant tilt angle between 0 and $\frac{\pi}{2} \cdot{ }^{2}$ In 2001 Dozov $^{3}$ when analysing the second-order elastic energy in a nematic phase composed of achiral "banana-shaped" molecules showed that two solutions may occur, a periodic oscillation of the local bend or a continuous conical twist-bend strucutre. The latter is twofold degenerate permitting left and right handed twist. Both 
solutions were proved to be energetically stable and could appear in mesogens with realistic molecular parameters. Independently, Memmer ${ }^{4}$ carried out Monte-Carlo simulations in molecular systems composed of banana shaped molecules and discovered that, near the transition to a highly ordered smectic phase, a nematic phase with hints of achiral symmetry breaking appears. This theoretical prediction sparked interest in banana-shaped molecules that may actually exhibit this new twist-bend phase $\left(\mathrm{N}_{t b}\right)$. Molecular dimers can behave like these bent shaped mesogens at relatively low temperatures and therefore be candidates for this phase. For instance, the liquid crystal dimer CB-C7-CB had already been reported in 1993, to have a high temperature uniaxial nematic phase followed by a lower temperature smectic phase. ${ }^{5}$ This was odd, considering that similar compounds only exhibited an uniaxial nematic phase. Taking this into account, the authors in ref. ${ }^{6}$ made a comprehensive study of this compound in order to verify if this intermediate phase was actually the twist-bend nematic phase, and arrived at results that agree with this interpretation. More recently, Robles-Hernández et al. ${ }^{7}$ focused on the study of the liquid crystal dimer system CB-C9-CB, which was also reported to have a nematic-nematic phase transition, ${ }^{8,9}$ and obtained similar results that also agree with the existence of periodically modulated structures. Papers ${ }^{6}$ and ${ }^{7}$ demonstrate, through deuterium NMR, that the two carbon-deuterium (CD) bonds at the terminal methylene groups of the linking chain loose their equivalence when entering the $\mathrm{N}_{t b}$ phase. The authors in ref. ${ }^{10}$ went even further and demonstrated that all the methylene groups in the linking chain of the CB-C7-CB compound in the $\mathrm{N}_{t b}$ phase have non equivalent bonds, except for the central group because of its two-fold symmetry. Other NMR studies using proton, deuterium and/or carbon 13, on $\mathrm{CBC} 7 \mathrm{CB},{ }^{11} \mathrm{CBC} 9 \mathrm{CB},{ }^{12-15}$ and similar compounds ${ }^{16}$ have been reported in the literature. These studies confirmed the uniaxial character of the $\mathrm{N}_{t b}$ phase.

An alternative model for the molecular organization in the low temperature nematic phase (labeled Nx) exhibited by these materials, supported on deuterium NMR measurements, has been proposed. ${ }^{17}$ The authors allege that if the helical distribution of the director was present 
in the Nx phase, then the deuterium spectrum, after a sample rotation of $90^{\circ}$ about an axis perpendicular to the magnetic field, should be a superposition of peaks corresponding to the continuous helix. Instead what they obtained was a typical spectrum of the perpendicular orientation relative to the magnetic field of a uniformly oriented director. According to this model, the molecules are arranged into small chiral clusters with opposite chirality giving rise to a phase structure with a global common director. However, the global heli-conical structure, corresponding to the $\mathrm{N}_{t b}$ phase could still be compatible with the deuterium data presented in ${ }^{17}$ if it is assumed that the molecular diffusion is fast enough regarding the NMR observation time. This interpretation is expanded in. ${ }^{18}$

The molecular organization in the $\mathrm{N}_{t b}$ phase, according to ${ }^{6}$ is similar to that found in related crystalline systems determined in the bulk by XRD studies ${ }^{19}$ or by the characterization of thin films by means of AFM. ${ }^{20}$ In recent studies, the chiral arrangement in the $\mathrm{N}_{t b}$ phase was probed by resonant X-ray diffraction giving evidence for an helical pitch of the order of $10 \mathrm{~nm}$, corresponding to two or three molecular lengths. ${ }^{21,22}$

In this work we present a Proton and Deuterium NMR study of the molecular orientational order in the liquid crystal dimer system 1",9"-bis(4-cyanobiphenil-4'-yl) nonane, usually labeled as CB-C9-CB. A numerical model that, for the first time, is able to simulate both the ${ }^{1} \mathrm{H}$ NMR spectra in conjunction with the deuterium splittings reported in ${ }^{17}$ is proposed, allowing the determination of the Saupe order matrix for the mesogenic molecular units. A detailed discussion of our results and their implications on the molecular orientational order of this system is presented. It specifically addresses the origin of the unusual dipolar splitting narrowing on decreasing the temperature in the $\mathrm{N}_{t b}$ phase and the double quadrupolar splitting observed from the first deuterated positions in the molecular chain found in this phase.

The detailed simulations presented herein show that the most ordered molecular frame (eigenframe of the Saupe tensor) ${ }^{23}$ changes its orientation on crossing the $\mathrm{N}-\mathrm{N}_{t b}$ phase transition. The orientation observed in the $\mathrm{N}_{t b}$ phase is compatible with a twisted conformation 
of the mesogenic units. This is clearly compatible with the onset of the helical structure of the $\mathrm{N}_{t b}$ phase, at least at a local level.

The layout of the paper is as follows: In section 'Experimental Details' we present and discuss the experimental details concerning the studied system and the ${ }^{1} \mathrm{H}$ NMR data acquired. In section 'Model' we describe the model developed for the proton NMR spectra. In section 'Data and Model Results' we discuss the results obtained with the prosed model. Finally, concluding remarks are summarized in section 'Final Remarks'.

\section{EXPERIMENTAL DETAILS}

The material considered in this work is the symmetric liquid crystal dimer 1",9"-bis(4cyanobiphenil-4'-yl) nonane, CB-C9-CB, presented in figure 1. This compound belongs to the $\mathrm{CB}-\mathrm{C} n-\mathrm{CB}$ series which consists of two cyanobiphenil $(\mathrm{CB})$ mesogenic units linked by a flexible alkyl chain with $n$ carbon atoms. This compound was synthesized in the department of chemistry of the University of Hull in the United Kingdom,. ${ }^{9}$ The phase sequence was determined using polarizing optical microscopy. The recorded transition temperatures are in good agreement with those presented in ${ }^{7}$ and. ${ }^{9}$

The ${ }^{1} \mathrm{H}$ NMR spectra were recorded on a Bruker $300 \mathrm{MHz}$ spectrometer, which has a magnetic field of 7.04T, equiped with a Bruker Avance II console. The sample was vaccum sealed in a tube with $5 \mathrm{~mm}$ in diameter. The absortion spectra was measured using a $90^{\circ}$ pulse width of $6 \mu s$. The recyling delay was set at $4 \mathrm{~s}$. For each temperature 256 free induction decay signals, FIDs, were recorded.

The sample was heated until it reached the isotropic liquid phase, which in the high magnetic field happened at $399.1 \pm 0.8 \mathrm{~K}$, indicating that the magnetic field induces some

order in the system as reported in ref. ${ }^{24}$ for this and similar systems. After the sample stabilised in this phase, the NMR spectra were acquired in a temperature sweep from 398 $\mathrm{K}$ to $339.3 \mathrm{~K}$ with a temperature step of approximately $2 \mathrm{~K}$. The sample temperature was 


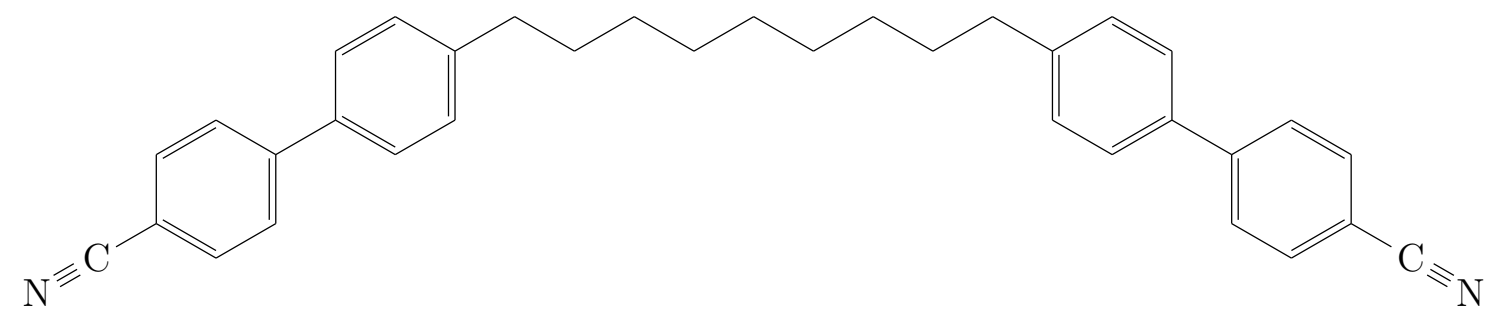

$$
\begin{aligned}
& \mathrm{N}_{t b} \rightarrow 380.1 \pm 0.2 \mathrm{~K} \mathrm{~N} \rightarrow 395.7 \pm 0.2 \mathrm{~K} \mathrm{Iso} \\
& \mathrm{Iso} \rightarrow 395.3 \pm 0.2 \mathrm{~K} \mathrm{~N} \rightarrow 379.5 \pm 0.2 \mathrm{~K} \mathrm{~N}_{t b}
\end{aligned}
$$

Figure 1: (a) Structure of the CB-C9-CB molecule. (b) Phase sequence obtained using polarising optical microscopy, POM.

controlled by an Eurotherm temperature controller.

\section{MODEL}

The Saupe order matrix characterizes the orientational order of rigid molecular segments in uniaxial phases, ${ }^{23}$ in our case, the Saupe order matrix for the mesogenic units is evaluated in both the $\mathrm{N}$ and $\mathrm{N}_{t b}$ phases using proton and deuterium spectra recorded over the temperature range studied. The determination of the Saupe order matrix for the CB-C9CB mesogenic units is based on a detailed simulation of the proton NMR spectra from a fully protonated sample along with an additional contribution from published data on the quadrupolar splittings obtained from a deuterated sample with deuterated positions in the first methylene groups of the flexible chain. ${ }^{17}$ The Saupe order matrix was determined for the molecular segment composed of the first methylene group of the chain and the two benzene rings of each mesogenic unit, considering the approximation that the different conformers of the mesogenic unit are obtained solely by $\pi$ flips of the benzene rings and thus share the same Saupe order matrix. It is also considered that the two halves of the molecule are NMR equivalent. An approximate simulation of the proton spectrum arising from one half of the molecular chain is also included in the spectra simulation.

The high field proton NMR spectra in liquid crystalline systems are dominated by the direct dipolar interaction between pairs of protons, due to the fast molecular self diffusion 
and the $1 / \mathrm{r}^{3}$ decay of the dipolar interaction, the monodomain proton spectrum is identical to that of a single LC molecule. The proton spin system of each molecule still includes a large number of spins and further approximations must be considered to reduce the dimension of the associated Hilbert Space to a workable size. The molecule CB-C9-CB is composed of two identical mesogenic parts linked by a flexible alkyl chain with an odd number of methylene groups. The NMR results published so far on similar compounds and our own data sets indicate that the two identical halves of the molecule are NMR equivalent. Hence simulation can be simplified by considering a molecular half. Each half is composed of a mesogenic group and of half of the flexible alkyl chain, with the central chain methylene group separating the two molecular halves. Thus, in our simulation of the proton spectrum, each half molecule is divided into 3 interacting spin groups. The first group is composed of the mesogenic unit and the adjacent first methylene group of the chain. The second one is composed of the flexible hydrocarbon (half)chain (three methylene groups). The third one includes the central methylene group of the chain (see figure 1). The reason for this approach is to reduce the dimension of the Hilbert space associated with the spin groups or, in other words, make the analysis possible. The spectrum is a sum of the contributions from each one of these three groups. The relevant NMR spin Hamiltonian for the spectrum evaluation from each interacting proton spin group includes 4 terms as follows. ${ }^{25}$

$$
\overline{\mathcal{H}}=\overline{\mathcal{H}}_{Z}+\overline{\mathcal{H}}_{\sigma}+\overline{\mathcal{H}}_{J}+\overline{\mathcal{H}}_{D}
$$

where $\mathcal{H}_{Z}$ is the Zeeman term, $\mathcal{H}_{\sigma}$ is the chemical shift, $\mathcal{H}_{J}$ is the indirect spin coupling and $\mathcal{H}_{D}$ the direct dipolar interaction. In our case, as we are analyzing the proton spectra from anisotropic LC phases the indirect spin coupling term $\overline{\mathcal{H}}_{J}$ is small in face of the direct dipolar interaction term $\overline{\mathcal{H}}_{D}{ }^{26}$ and will be neglected in our analysis. In high field NMR spectroscopy only the secular part of the Hamiltonian is relevant leading to the form ${ }^{25}$ 


$$
\begin{aligned}
\overline{\mathcal{H}}_{Z}+\overline{\mathcal{H}}_{\sigma} & =-\sum_{k=1}^{n} \gamma \hbar I_{k, z}\left(1-\bar{\sigma}_{k, z z}\right) B_{0} \\
\overline{\mathcal{H}}_{D} & =\frac{\mu_{0}}{4 \pi} \sum_{k=1, l>k}^{n-1, n}\left\{\left[I_{k, z} I_{l, z}-\frac{1}{4}\left(I_{k,+} I_{l,-}+I_{k,-} I_{l,+}\right)\right] \overline{\frac{\gamma^{2} \hbar^{2}}{r_{k l}^{3}}\left(1-\frac{3}{r_{k l}^{2}} r_{k l, z}^{2}\right)}\right\},
\end{aligned}
$$

where $\gamma$ is the gyromagnetic ratio for protons, $\hbar$ is the Plank's constant divided by $2 \pi, I_{i, j}$ is $\mathrm{j}$ component of the angular momentum spin operator for spin $\mathrm{i}, B_{0}$ is the static magnetic induction defining the $\mathrm{z}$ lab. frame axis, $\mu_{0}$ is the vacuum magnetic permeability. The quantities $\bar{\sigma}_{k, z z}$ and $\bar{D}_{k l, z z}$ with

$$
\bar{D}_{k l, z z} \equiv \frac{\mu_{0}}{8 \pi^{2}} \overline{\frac{\gamma^{2} \hbar}{r_{k l}^{3}}\left(1-\frac{3}{r_{k l}^{2}} r_{k l, z}^{2}\right)},
$$

are the zz components in the laboratory frame of motionally averaged second rank tensorial quantities, respectively the chemical shielding tensor for spin $\mathrm{k}$ and the direct dipolar interaction tensor for spins $\mathrm{k}$ and $\mathrm{l}$. The quantities $\bar{D}_{k l, z z}$ can be related to the molecular defined tensors $D_{k l, z z}^{M}$ as follows

$$
\bar{D}_{k l, z z}=\sum_{i} p_{i} \frac{2}{3} S_{i, \alpha \beta}^{z z} D_{i, k l, \alpha \beta}^{M}
$$

where the sum is extended over the different conformers of the molecular segment, $p_{i}$ is the probability of conformer 'i', $S_{i, \alpha \beta}^{z z}$ is the Saupe order tensor of conformer 'i' and $D_{i, k l, \alpha \beta}^{M}$ is the direct dipolar iteration tensor in the molecular frame associated with conformer 'i'. Summation over repeated indices is implied. When the molecular segment is rigid or the order tensors for the different conformers are the same, one order matrix sets the relation between $\bar{D}_{k l, z z}$ and $D_{i, k l, \alpha \beta}^{M}$. This is the case for the mesogenic units where we have considered that the different conformers are generated by $\pi$ flips of the two benzene rings. In this case equation 4 becomes

$$
\bar{D}_{k l, z z}=\frac{2}{3} S_{\alpha \beta}^{z z} D_{k l, \alpha \beta}^{M}
$$


where $D_{\alpha \beta}^{M}$ is the direct dipolar interaction tensor in the molecular frame. A similar relation could be considered for the tensorial quantities $\bar{\sigma}_{k, z z}$ but their dependence on the magnetic susceptibility of the NMR probehead ${ }^{27}$ make them more difficult to simulate. Introducing the eigenframe of the Saupe order tensor 'p' and the order parameters $S \equiv S_{z z}^{z z, p}$ and $D \equiv$ $S_{x x}^{z z, p}-S_{y y}^{z z, p}$ the relation 5 can be cast in the form

$$
\bar{D}_{k l, z z}=S D_{z z}^{M, p}+\frac{D}{3}\left(D_{x x}^{M, p}-D_{y y}^{M, p}\right)
$$

where the quantities $D_{i j}^{M, p}$ are given by a similarity transformation in terms of the dipolar interaction tensor $D_{i j}^{M}$ in a preset molecular frame and the rotation matrix $R(\alpha, \beta, \delta)$ as follows

$$
D_{i j}^{M, p}=R_{i k}(\alpha, \beta, \delta) D_{k, l}^{M} R_{l j}^{T}(\alpha, \beta, \delta)
$$

The Euler angles $\alpha, \beta$ and $\delta$ define the orientation of the most ordered frame (eigenframe) of the Saupe order tensor in the preset frame ${ }^{28}$ according to figure 2. The order parameters

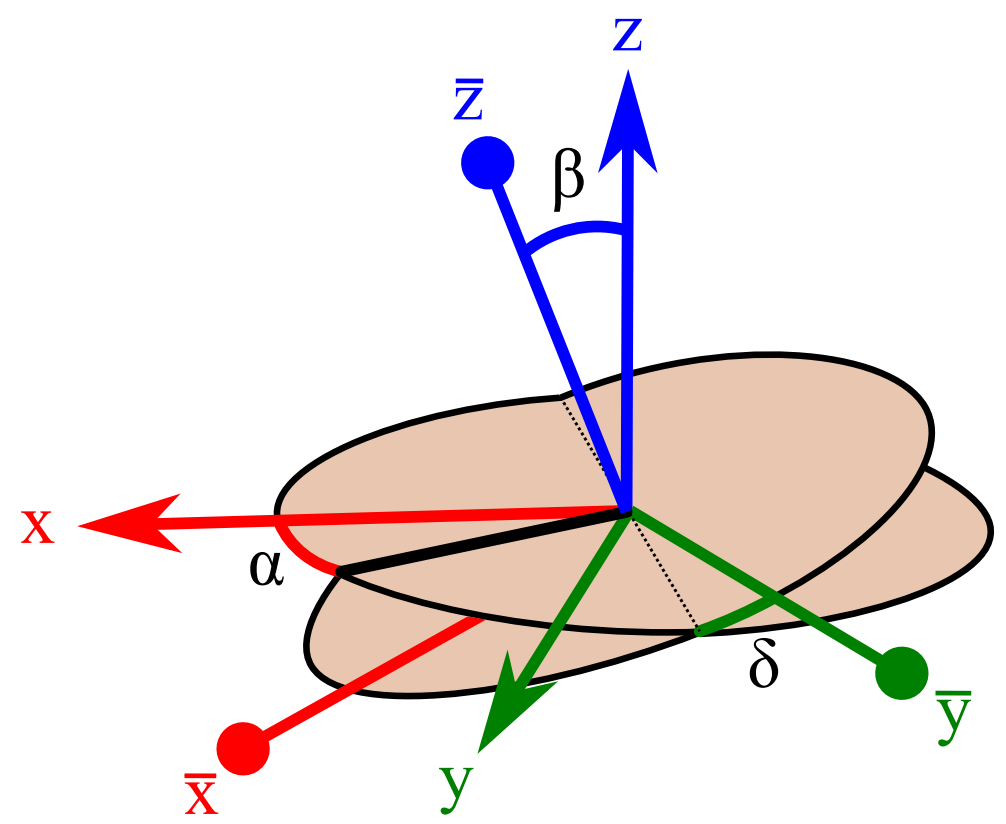

Figure 2: Representation of the Euler angles according to the convention defined in. ${ }^{28}$

$\mathrm{S}, \mathrm{D}$ and the angles $\alpha, \beta$ and $\delta$ associated with the mesogenic unit spin group are fitting parameters in the model. The preset molecular frame is chosen with its y-z plane perpen- 
dicular to the plane defined by the two $\mathrm{CH}$ bonds of the first methylene group of the chain and bisecting the angle between these two $\mathrm{CH}$ bonds, the y axis is chosen perpendicular to the plane defined by the $\mathrm{x}$ axis and the benzene rings rotation axis and the $\mathrm{z}$ axis is perpendicular to the $\mathrm{y}$ and $\mathrm{x}$ axis. The orientation of each benzene ring in the mesogenic group is parametrized by one angle between the normal to the ring plane and the $\mathrm{x}$ axis. The two angles corresponding to both benzene rings are fitting parameters, see figure 3 for details. For the remaining two spin groups, the half chain and the central methylene group,

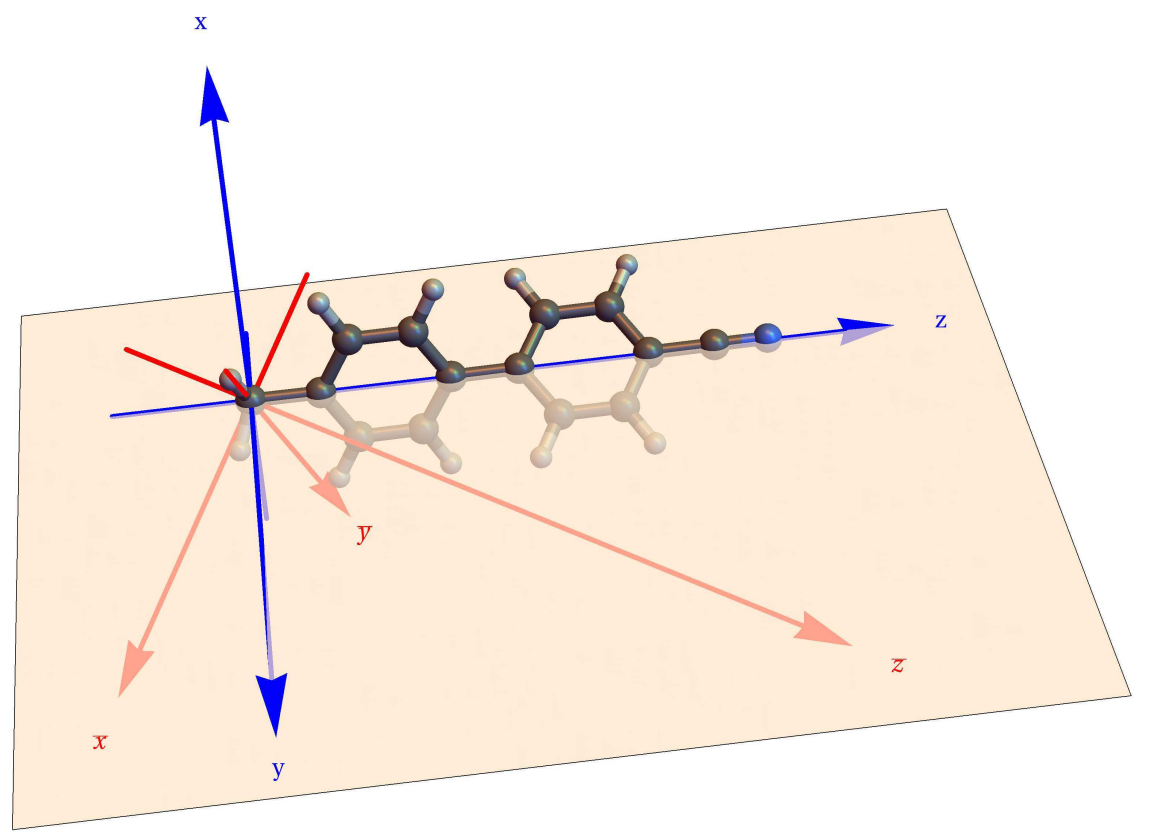

Figure 3: Ball and stick representation of the simulated system. The blue $(x y z)$ and red $(\bar{x} \bar{y} \bar{z})$ arrows define the selected and the most ordered molecular frames, respectively. The represented plane corresponds to the plane of symmetry defined by the two $\mathrm{CH}$ bonds of the first methilene group of flexible chain of the CB-C9-CB.

respectively, the averaged dipolar interaction tensors were evaluated assuming that there exists a dominant conformation corresponding to the all trans conformation of the methylene groups. The relations 5 to 7 for these groups were considered with the respective D order parameters set to 0 and the order parameters $\mathrm{S}$ for each group taken as fitting parameters. The two angles $\beta$ for these spin groups were set equal to the angle $\beta$ from the mesogenic unit, while the angle $\alpha$ of the central chain spin group was set to $\pi / 2$. This value was taken 
in order to maintain the two hydrogens NMR equivalent (producing equal NMR signals) at all temperatures. ${ }^{10}$ The angle $\alpha$ from the half chain spin group was set equal to $\alpha$ from the mesogenic unit.

For the averaged chemical shift tensor components $\bar{\sigma}_{k, z z}$ three independent fitting quantities were considered, one corresponding to the protons attached to the aromatic rings, other to the half chain protons and the third to the central methylene protons.

To build the spin Hamiltonian for each interacting spin group we must also obtain the spin operators respectively $I_{k, z}, I_{k,+}$ and $I_{k,-}$. This is achieved by expanding the spin operators for one spin to the dimension of the Hilbert space of the spin group using the tensor product. ${ }^{29}$ The spin Hamiltonian is then evaluated in the rotating frame through a unitary transformation. ${ }^{30}$

Next, the eigenvalues $\mathrm{E}_{i}$ and eigenvectors $\left|\psi_{i}\right\rangle$ of the Hamiltonian in the rotating frame are determined using a Jacobi routine ${ }^{31}$ and the simulated free induction decay after a $\pi / 2$ pulse follows as

$$
G(t)=c \sum_{j=1}^{N} \sum_{k>j}^{N} W_{j k} e^{-\left[i\left(\frac{E_{j}-E_{k}}{\hbar}\right)+L B_{j k}\right] t}
$$

with $W_{j k} \propto c\left|\left\langle\psi_{j}\left|I^{+}\right| \psi_{k}\right\rangle\right|^{232}$ and

$$
L B_{i j}=\sum_{k=1}^{n} \frac{X_{k i j} K_{K}}{\sum_{k=1}^{n} X_{k i j}},
$$

with $X_{k i j} \equiv\left|\left\langle\psi_{i}\left|I_{k}^{+}\right| \psi_{j}\right\rangle\right|^{2} . W_{j k}$ is zero unless the NMR selection rule $\Delta m= \pm 1^{25}$ is obeyed. In this case $\delta m=m_{j}-m_{k}=1$, where $\mathrm{m}_{j}$ and $\mathrm{m}_{k}$ are the magnetic quantum numbers of states $\mathrm{j}$ and $\mathrm{k} . \mathrm{N}=2^{n}$ is the number of spin states and $\mathrm{n}$ is the number of spins in the spin group, $K_{k}$ are line broadening fitting parameters for each spin in the spin group considered. The proton NMR absorption spectrum is obtained by Fourier transforming the simulated free induction decay with contributions from the 3 spin groups properly scaled to account for the number of spins in each group.

Quadrupolar splittings were also recorded for CB-C9-CB and reported by Hoffman et 
al. ${ }^{17}$ as referred above and their relation to the Saupe order tensor is detailed next. Due to the quadrupolar interaction, the quadrupolar splitting arising from a CD bond is given by

$$
\Delta \nu=\frac{3 e Q}{2 h} \overline{V_{z z}}
$$

where e is the elementary charge, $\mathrm{h}$ is Planck's constant, $\mathrm{Q}$ is the electric quadrupolar moment of the deuterium nucleus and $\overline{V_{z z}}$ is the average zz component of the electric field gradient tensor present at the nucleus site in the lab. frame. The quantity $\frac{e Q}{h} \overline{V_{z z}}$ can be related to the quantity $\nu_{Q} \equiv \frac{e Q}{h} V_{z^{\prime} z^{\prime}}$ through the Saupe order matrix of the rigid segment attached to the CD bond, the z' axis refers to an axis parallel to the CD bond. $\nu_{Q}$ is known from solid state and for sp3 bonds takes the value of $170 \pm 5 K \mathrm{~Hz}^{33}$ For an aligned sample with the director along the external magnetic field, the quadrupolar splitting arising from the $\mathrm{k}$ CD bond becomes

$$
\Delta \nu_{k}=\frac{e Q}{h} S_{\alpha \beta}^{Z Z} V_{k, \alpha \beta}^{M}
$$

In the most ordered frame 'p' the relation 11 takes the form

$$
\Delta \nu_{k}=\frac{3}{2} \nu_{Q}\left[S\left(\frac{3}{2} n_{k, Z p}^{2}-\frac{1}{2}\right)+\frac{D}{2}\left(n_{k, X p}^{2}-n_{k, Y p}^{2}\right)\right]
$$

where $n_{k, i p}=n_{k, i p}(\alpha, \beta, \delta)$ is the 'i' component of the $\mathrm{k}$ CD bond direction aligned unit vector in the 'p' frame (eigenframe of the Saupe order tensor), these quantities are functions of the angles $\alpha, \beta, \delta$ introduced above. In the $\mathrm{N}_{t b}$ phase two quadrupolar splittings arising from the non-equivalente CD bonds of the first methylene groups in the chain are observed experimentally, giving rise to two equations that merge into one in the $\mathrm{N}$ phase, these equations are linear in the order parameters $\mathrm{S}$ and $\mathrm{D}$. They are solved to obtain $\mathrm{S}$ and $\mathrm{D}$ for the mesogenic unit from the experimental quadrupolar splittings and the angles $\alpha, \beta$ and $\delta$. As the values for the biaxiality parameter are generally very low, we also considered the angle $\delta$ that minimised $D$. 


\section{DATA AND MODEL RESULTS}

The aim of our simulation of the proton spectra, along with the quadrupolar splittings reported in, ${ }^{17}$ of this liquid crystalline system was the determination of the Saupe order tensor for the mesogenic groups, in the hope of drawing some conclusions regarding the molecular orientational order. The acquired proton NMR spectra are represented in figure 4a. In figure $4 \mathrm{~b}$ we represented the deuterium splittings reported in ${ }^{17}$ and included interpolation results that matched the temperatures of our proton spectra.

Careful examination of the proton spectra reveals that it varies continuously with temperature. The line splittings increase in the high temperatures of the uniaxial nematic phase, however, at approximately $T=387 K$ the line splittings start decreasing. This decrease appears to be approximately monotonous with decreasing temperature. Regarding the quadrupolar splittings, it is evident that in the onset of the $\mathrm{N}_{t b}$ phase, the carbon deuterium bonds loose their equivalence since the quadrupolar splittings cease to be equal. The difference between the two quadrupolar splittings increases with decreasing temperature.

For each proton spectra inside the valid interpolation region of the quadrupolar splittings reported in, ${ }^{17}$ which includes one spectral point in the $\mathrm{N}$ phase and seventeen points in the $\mathrm{Nx} / \mathrm{N}_{t b}$ phase, we used our model to simulate the spectra. A selection of the obtained results, including one spectral point in the $\mathrm{N}$ phase is shown in figure 5 for the assignment $\left|\nu_{q, 1}\right| \geq\left|\nu_{q, 2}\right|$ and in figure 6 for $\left|\nu_{q, 1}\right| \leq\left|\nu_{q, 2}\right|$. The simulations for both assignments fit the data well and the difference between assignments is almost negligible. The main regions where the difference between the experimental data and the simulations is noticeable are in the deeps in the low negative frequencies, where the spectra are appreciably asymmetric.

The results presented in figures 5 show that the biggest contribution to the profile of the spectra comes from the mesogenic group, while the flexible chain predominantly accounts for the lower frequencies and the deeps at higher frequencies. The contribution of the central methylene group in the spectrum is essentially in the fine details of the more intense peaks.

In figures 7 and 8 we present the evolution of the molecular parameters with tempera- 
(a)
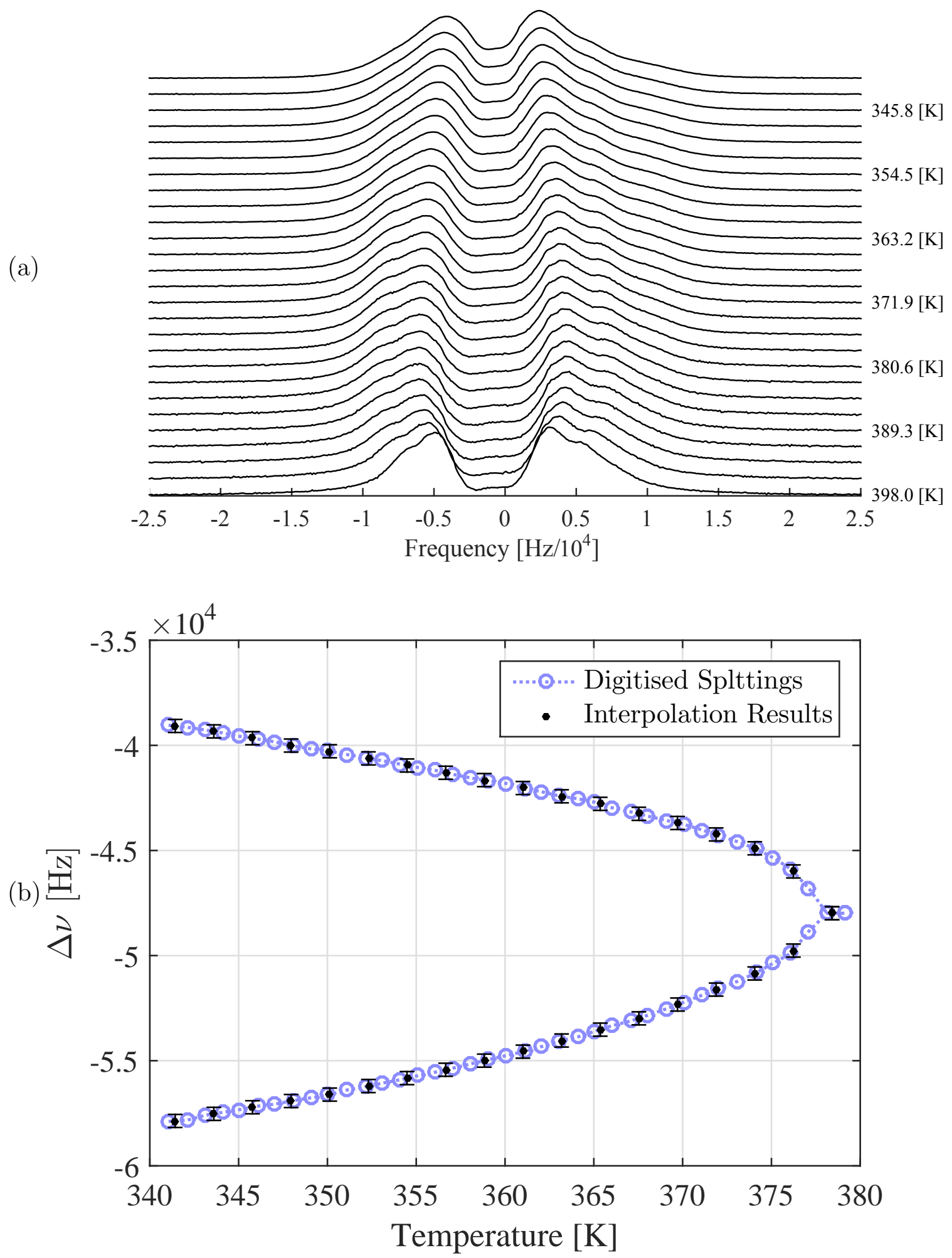

Figure 4: NMR data of the CB-C9-CB dimer system. (a) Stack plot representation of the ${ }^{1} \mathrm{H}$ NMR spectra, recorded on a cooling run. (b) Deuterium splittings published in, ${ }^{17}$ digitised results (०), interpolation results (•). 
ture, namely the order parameters $\mathrm{S}$ and $\mathrm{D}$ and the orientation angles $\alpha, \beta$ and $\delta$ for both assignments, $\left|\nu_{q, 1}\right| \geq\left|\nu_{q, 2}\right|$ and $\left|\nu_{q, 1}\right| \leq\left|\nu_{q, 2}\right|$.

Regarding the molecular order parameter, we observe that the values remain almost constant, fluctuating between $\sim 0.44$ and $\sim 0.47$ with a maximum deviation from its average of $\sim 3 \%$ for the assignment $\left|\nu_{q, 1}\right| \geq\left|\nu_{q, 2}\right|$ and $\sim 1.8 \%$ for $\left|\nu_{q, 1}\right| \leq\left|\nu_{q, 2}\right|$. Hence, we conclude that in this temperature region, the evolution of the order parameter is not responsible for the change in the profile of the ${ }^{1} \mathrm{H}$ and ${ }^{2} \mathrm{H}$ NMR results. The results we obtained for $S$ are of relatively small values considering the related system $5 \mathrm{CB}$, for which $S \sim 0.52$ has been reported, at $T=T_{C}-9 \mathrm{~K}$, where $T_{C}$ is the clearing temperature. ${ }^{34}$ Since the chemical structure of the $5 \mathrm{CB}$ molecule is similar to the molecular segment we simulated, this indicates that the bent structure of CB-C9-CB induces packing constraints that reduce the value of the order parameter, $S$, of the system.

The molecular biaxiality parameter $D$ is null in the $\mathrm{N}$ phase and, with decreasing temperature it increases if $\left|\nu_{q, 1}\right| \geq\left|\nu_{q, 2}\right|$ and decreases if $\left|\nu_{q, 1}\right| \leq\left|\nu_{q, 2}\right|$ until it stabilises at approximately $T=363.2 \pm 0.8 \mathrm{~K}$. It is noteworthy to point out that its evolution is symmetric with respect to the axis defined by $D=0$. This means that, if we consider $\left|\nu_{q, 1}\right| \geq\left|\nu_{q, 2}\right|$, the director distribution function in the molecular frame is predominant in the vicinity of the $x O z$ plane of the most ordered frame while if $\nu_{q, 1}|\leq| \nu_{q, 2} \mid$ it is predominant in the vicinity of the $y O z$ frame. This result is concordant with the hypothesis that the molecules pack themselves in twisted and bent domains with opposing chirality. The helical ordering of the mesogens with a tilt of the aromatic groups at an angle $\beta$ is responsible for the measured molecular biaxiality.

Regarding the angular parameter $\beta$, which describes the angle between the most ordered molecular frame's $z$ axis and the $z$ axis of the molecular frame we defined, it increases almost linearly with decreasing temperature. This behaviour matches the results we obtained in ${ }^{35}$ with an approximate description of $\beta$. The angles $\alpha$ and $\delta$ exhibit a symmetric evolution between assignments. It is also worth mentioning that these two parameters together with 
$D$ stabilise at about $T=365 \mathrm{~K}$, while $\beta$ continues to increase as temperature decreases. It is clear that the angular parameters together with $D$ are the main mechanism that causes the change of profile in the spectra. In figure 9, the evolution of the molecular frame (see figure 3) relative to the most ordered molecular frame (eigenframe of the Saupe tensor) with decreasing temperature is shown. In both assignments we can observe that the $z$ axis of the most ordered frame tilts significantly away from the para axis of the benzene rings, and that, as soon as we enter the $\mathrm{N}_{t b}$ phase, this axis leaves the plane of symmetry of the carbonproton bonds of the first element in the linking chain, causing the loss of NMR equivalence of those bonds. In other words, this represents the first direct experimental evidence that the methylene groups in the linking chain are not in the all-trans conformation, in accord with the proposed twisted conformation of the molecules in the $\mathrm{N}_{t b}$ phase. The angle between the $\mathrm{z}$ axis of the most ordered molecular frame and its projection on the symmetry plane of the carbon-proton bonds of the first element in the linking chain, changes from zero in the $\mathrm{N}$ phase to values in the range of 0.009 to $0.3 \mathrm{rad}$ in the $\mathrm{N}_{t b}$ phase, according to the results presented in figure 8. This departure from zero translates the loss of achiral symmetry giving rise to the chiral $\mathrm{N}_{t b}$ phase. This behaviour is comparable to the one described in reference ${ }^{36}$ where the evolution of the Saupe order tensor is studied going from a non-chiral racemic system to a chiral one. The biaxiality parameter $D$ and the angular parameters $\alpha, \beta, \delta$ shown in figures 7,8 and $8 \mathrm{~b}$ all exhibit significantly larger uncertainties in the $\mathrm{T}=375 \mathrm{~K}$ to $\mathrm{T}=365 \mathrm{~K}$ temperature range. This increase is a result of numerical indeterminations in the least squares minimisation algorithm.

In section 'Model' we mentioned that the parameter $\delta$ was obtained minimising $D$. In order to ensure that this condition was not being too restrictive we relaxed it and fitted the experimental data in some representative temperatures. The results we obtained matched the minimums we had obtained before and did not resulted in a significant decrease in the $\chi^{2} /$ n.d.f. (n.d.f=number of experimental points - number of fitting parameters), which indicates that our results, with $D$ minimized, are compatible with the best $\chi^{2} /$ n.d.f.. 
$371.9 \mathrm{~K}$
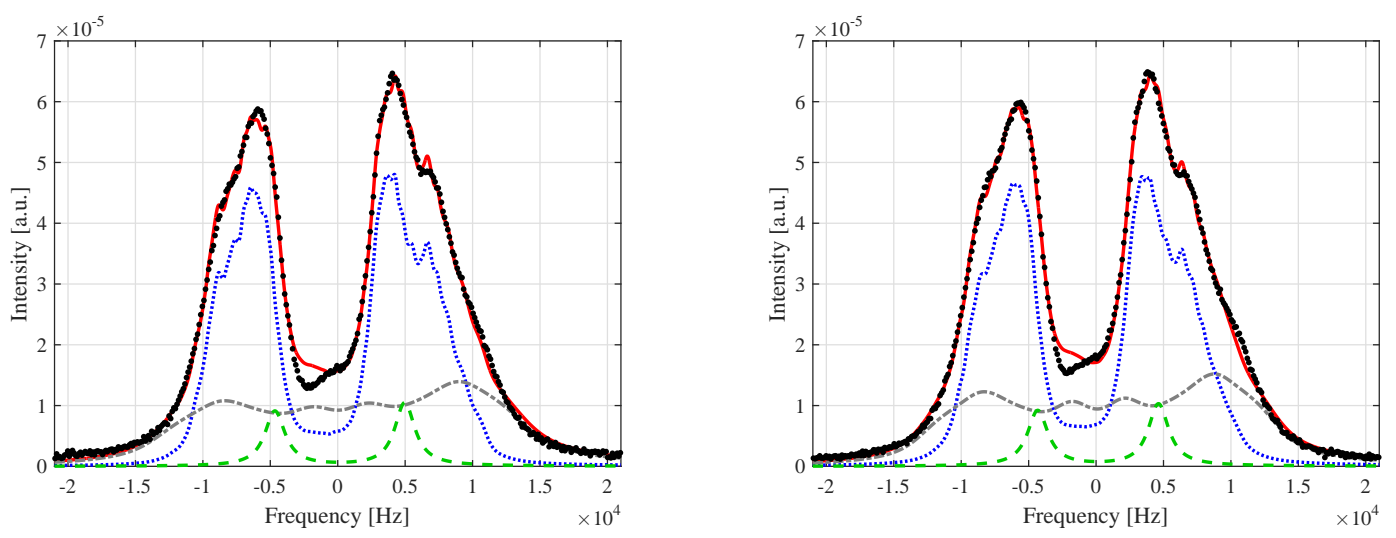

$358.8 \mathrm{~K}$
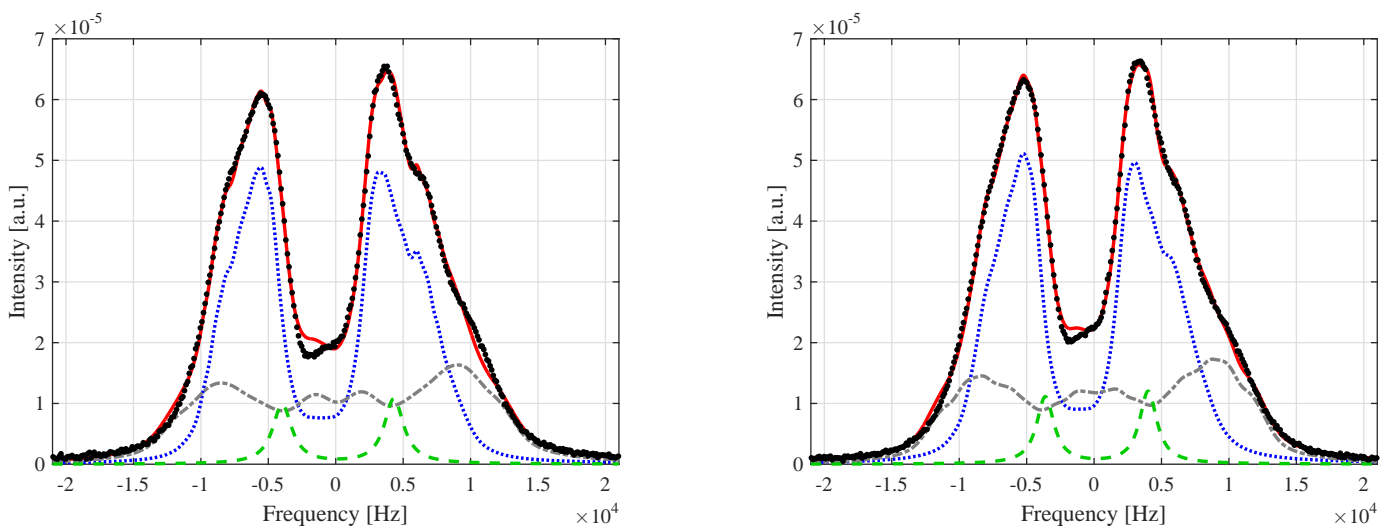

$341.4 \mathrm{~K}$
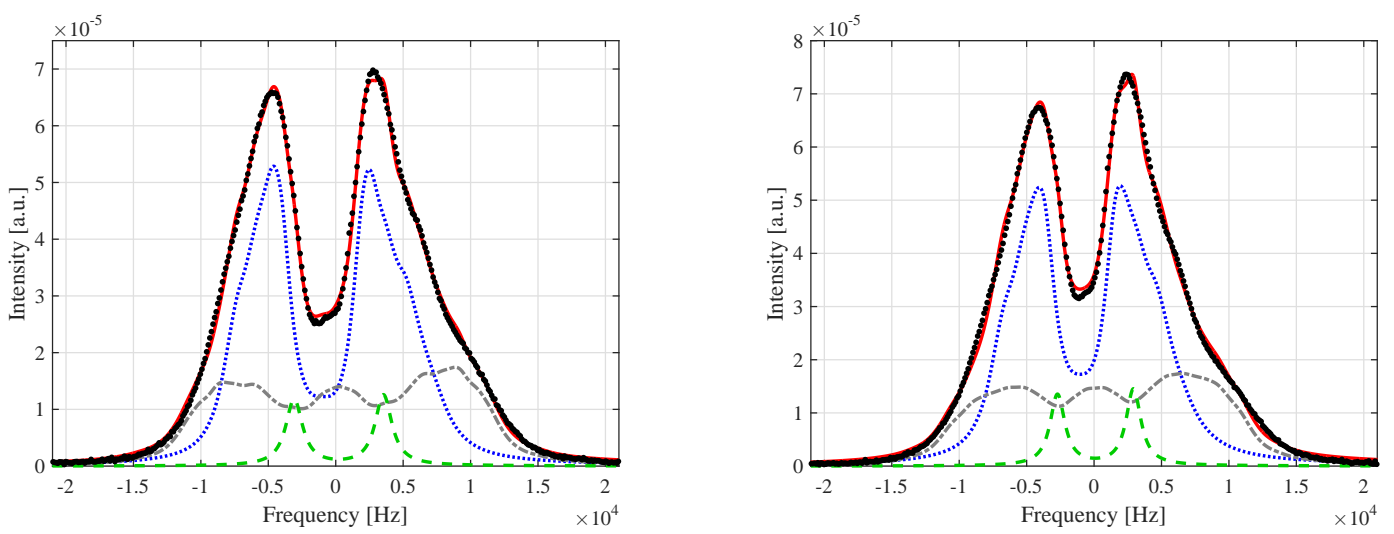

Figure 5: Representative results for the assignment $\left|\nu_{q, 1}\right| \geq\left|\nu_{q, 2}\right|$. Experimental data (•) and simulation results (-) along with the contribution of the different molecular groups: mesogenic group contributions (..), flexible chain (---) and central methylene (- -) were included. 
$371.9 \mathrm{~K}$
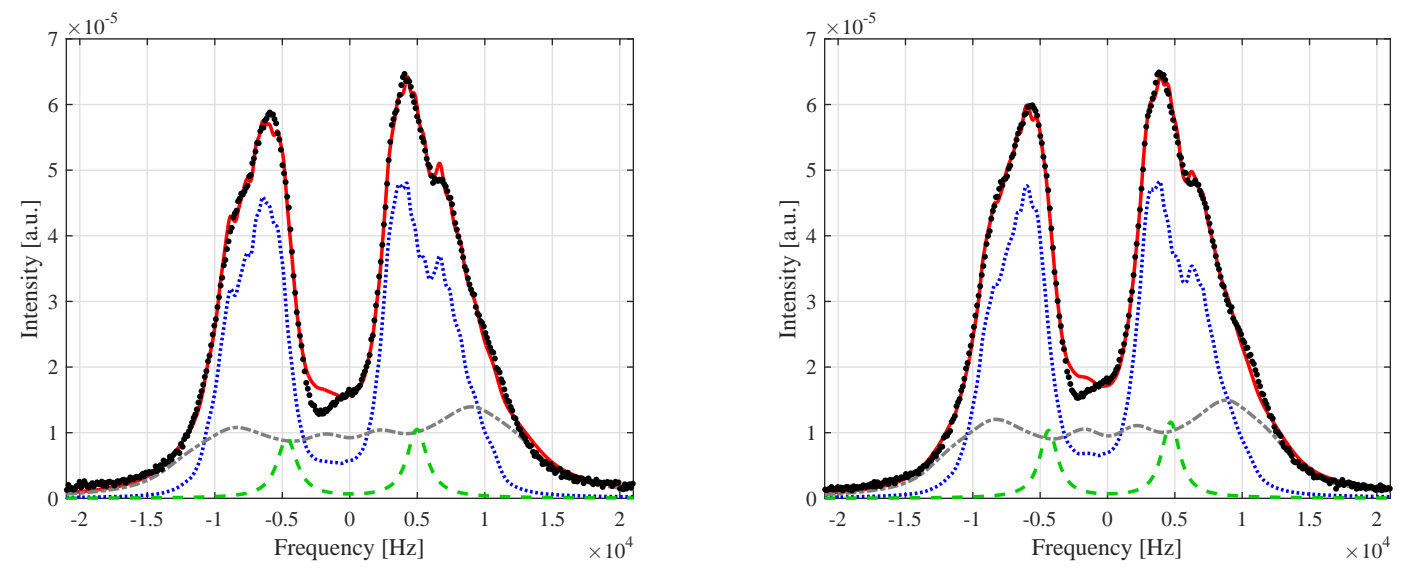

$358.8 \mathrm{~K}$
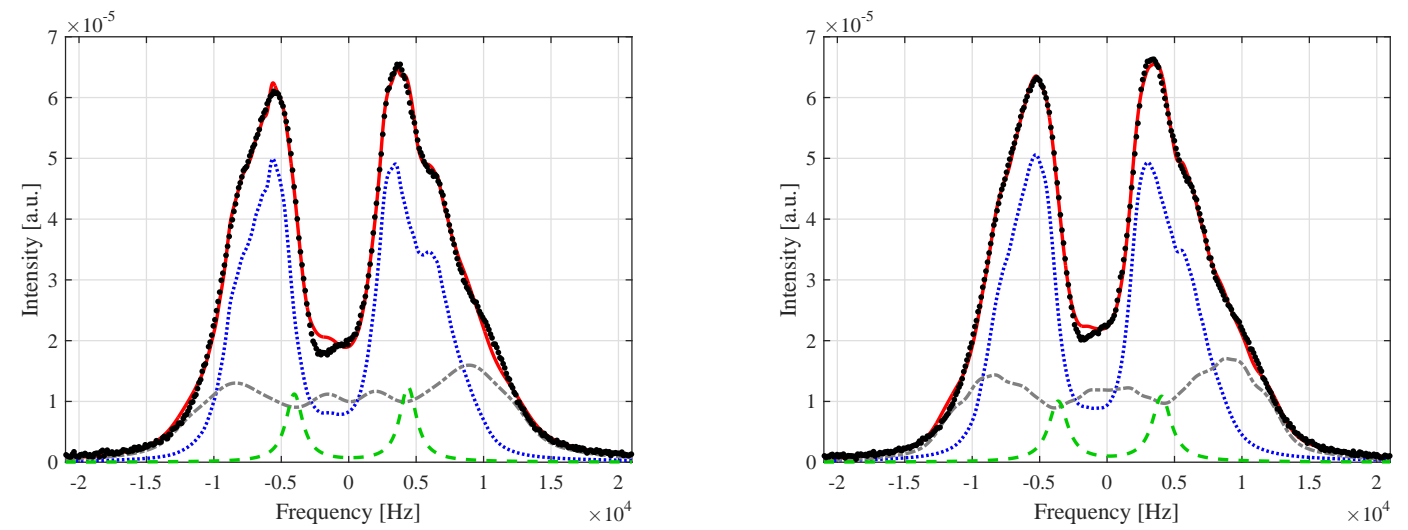

$341.4 \mathrm{~K}$
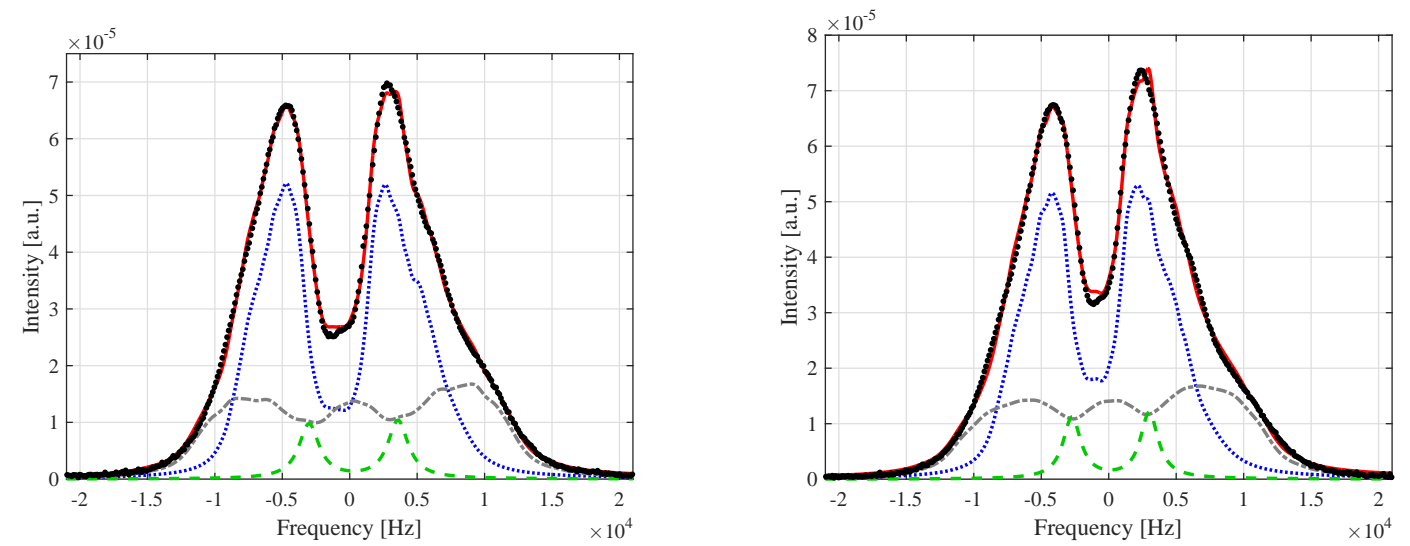

Figure 6: Representative results for the assignment $\left|\nu_{q, 1}\right| \leq\left|\nu_{q, 2}\right|$. Experimental data along with the contribution of the different molecular groups was represented in the same way as in figure 5. 


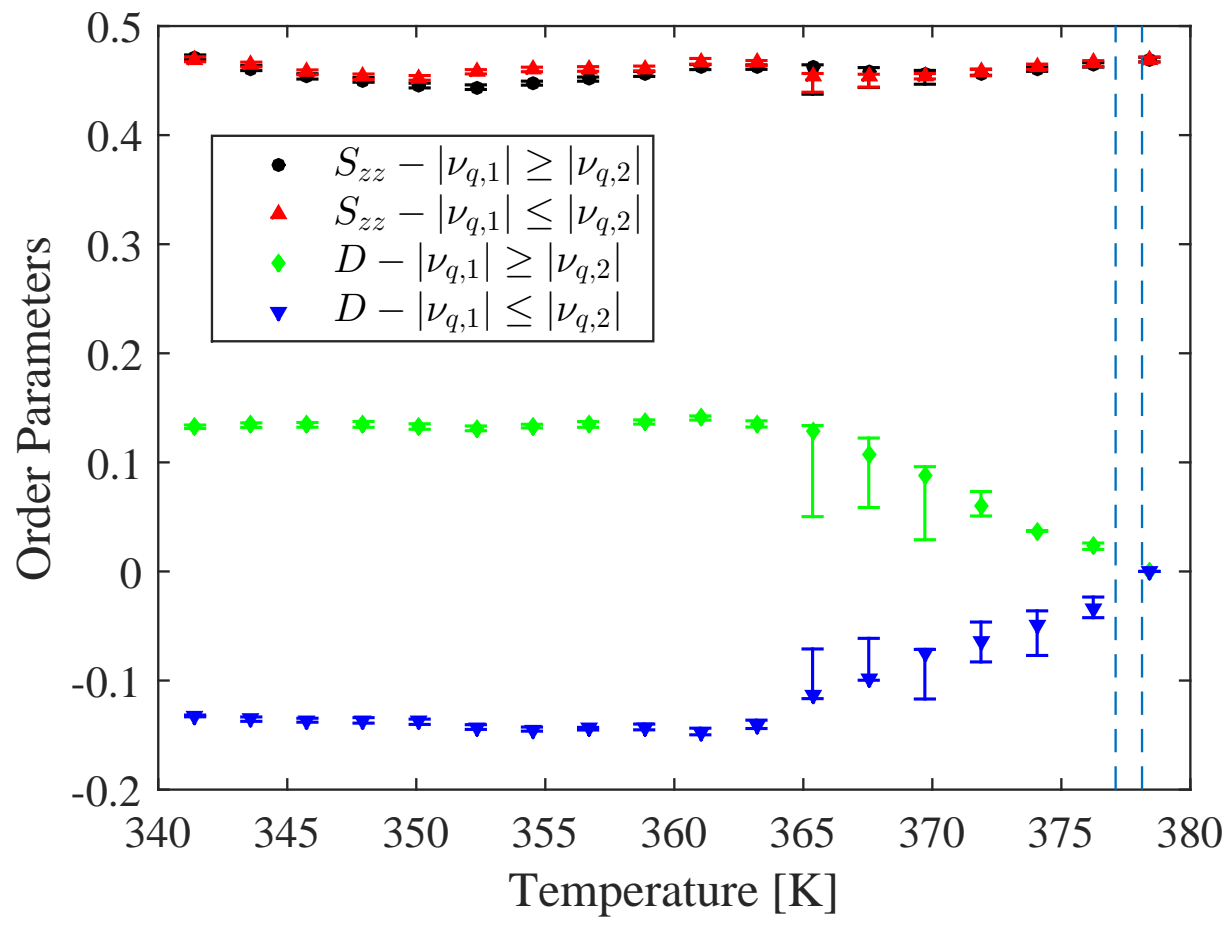

Figure 7: Molecular order parameter and molecular biaxialiaty parameter as a function of temperature for both simulated assignments. Dashed blue lines indicate the temperature range at which the quadrupolar line splittings reported in ${ }^{17}$ loose their equivalence. 

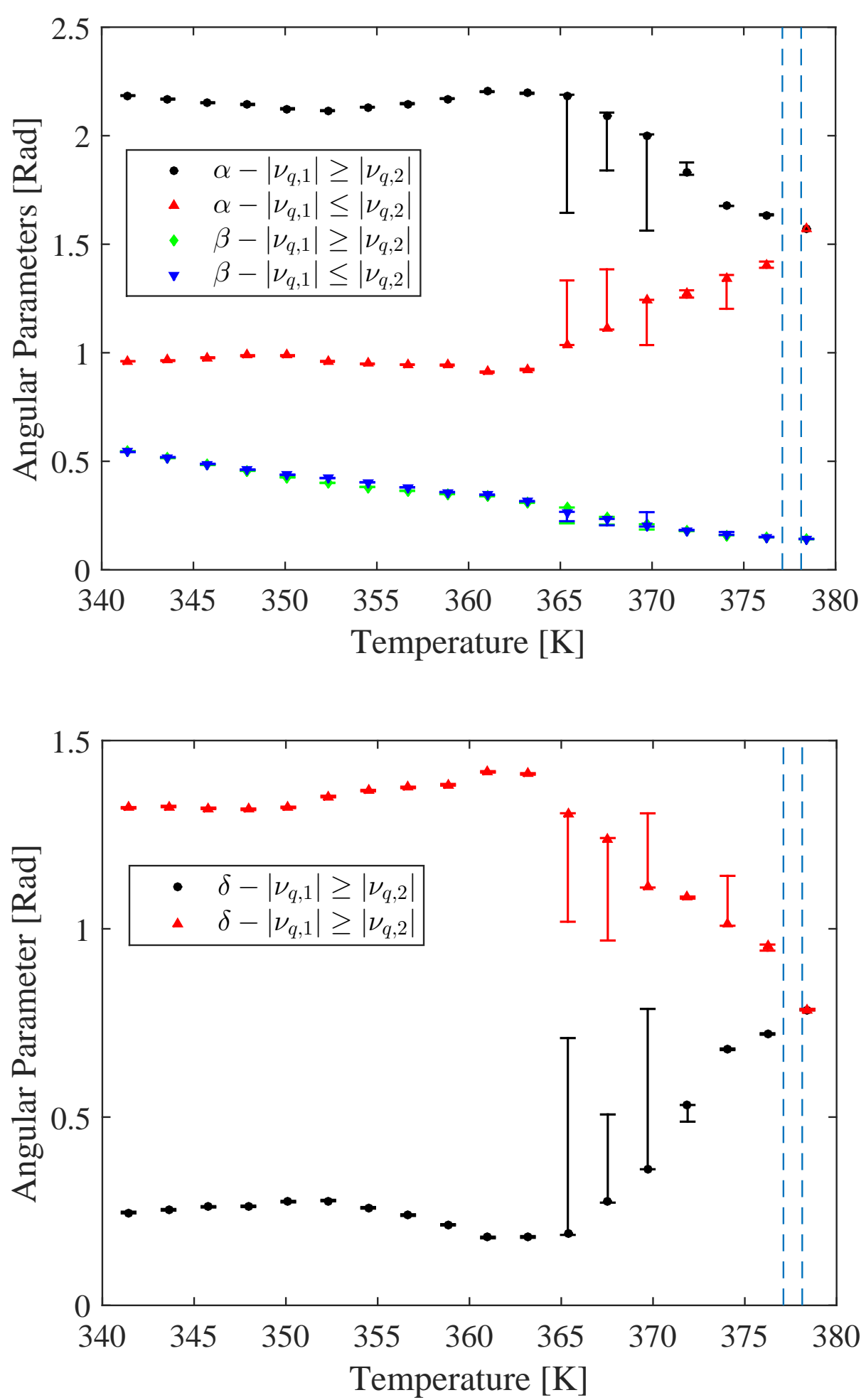

Figure 8: Angular parameters defined according to figure 2, that define the orientation of the most ordered molecular frame with respect to a selected molecular frame, as a function of temperature, see figure 3. Dashed blue lines indicate the temperature range at which the quadrupolar line splittings reported in ${ }^{17}$ loose their equivalence. 


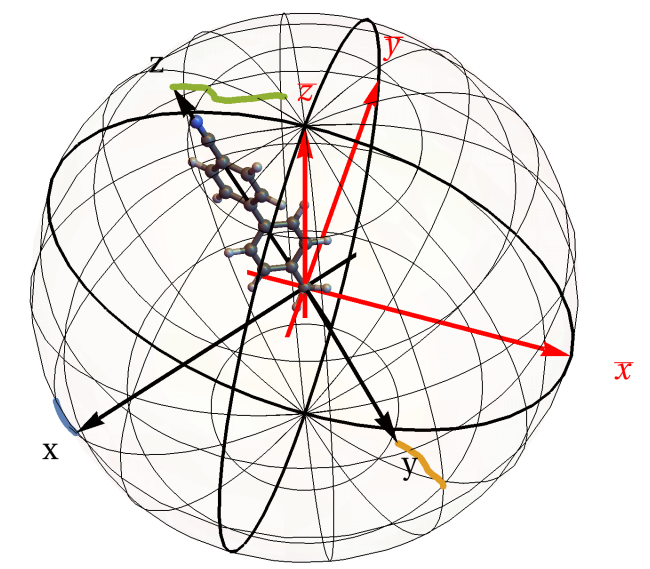

(a) Assignment $\left|\nu_{q, 1}\right| \geq\left|\nu_{q, 2}\right|$

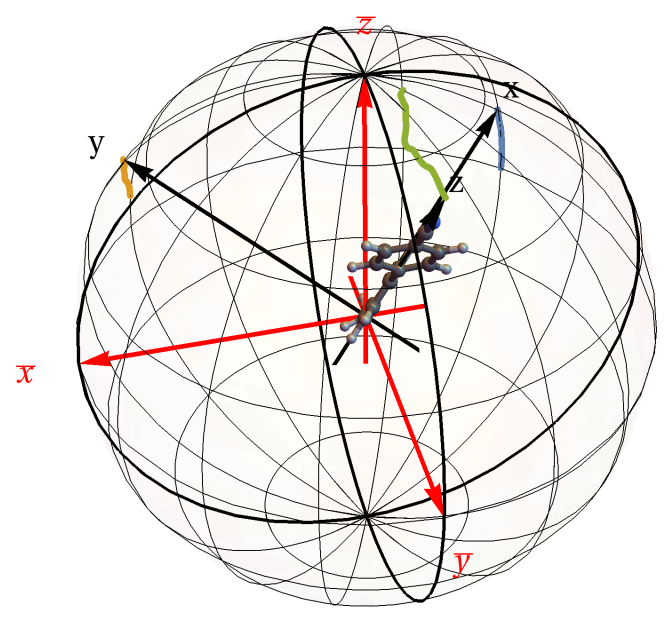

(b) Assignment $\left|\nu_{q, 1}\right| \leq\left|\nu_{q, 2}\right|$

Figure 9: Representation of the evolution of selected molecular frame (defined by $(x, y, z)$ and represented in black) in the most ordered molecular frame (defined by the axis $(\bar{x}, \bar{y}, \bar{z}$ ) and represented in red), for each assignment with decreasing temperature. The represented conformation of the molecule's mesogenic group corresponds to $T=341.4 \pm 0.8 \mathrm{~K}$ and the curves in blue, orange and green define the evolution of each axis. 


\section{CONCLUDING REMARKS}

In summary, we have presented a NMR spectroscopy study of the CB-C9-CB liquid crystal dimer system. We focused on the description of the molecular orientational order of this system in the $\mathrm{N}$ and $\mathrm{N}_{t b}$ phases using a numerical model which, for the first time, describes in detail the ${ }^{1} \mathrm{H}$ NMR spectra in conjunction with the deuterium NMR data reported in. ${ }^{17}$ This study allows for a precise characterization of the local molecular orientation in the $\mathrm{N}_{t b}$ phase. This results are an important step for a complete description of the $\mathrm{N}_{t b}$ phase structure.

This investigation show that the order parameter $S$ of the mesogenic group remained almost constant, in the studied temperature range. This result is compatible with our previous interpretation presented in ${ }^{35}$ since, according to the Haller law, the order parameter shows a small variation for values far below the N-I transition temperature. Our simulations indicate that main mechanism inducing the change of the proton and deuterium NMR spectra is the change in the orientation of the most ordered molecular frame relative to the selected molecular frame along with the change of molecular biaxiality (given by D parameter) varying from zero in the $\mathrm{N}$ phase to a finite value, well above the experimental error, in the $\mathrm{N}_{t b}$ phase.

The angle $\beta$ between the most ordered molecular frame's $z$ axis (parallel to the nematic director) and the para-axis of the benzene rings increases approximately linearly with decreasing temperature. This behaviour matches the results obtained in. ${ }^{35}$ This study also shows that, at the $\mathrm{N}-\mathrm{N}_{t b}$ phase transition, the $z$ axis of the most ordered molecular frame leaves the plane of symmetry that bisects the angle defined by the two $\mathrm{CH}$ bond in the first position of the linking chain of the dimer (see figure 3).

The spectra were simulated considering two possible assignments, $\nu_{q, 1}|\geq| \nu_{q, 2} \mid$ and $\nu_{q, 1} \mid \leq$ $\left|\nu_{q, 2}\right|$ (see figure 3). When comparing the results for both assignments, we conclude that

they are mutually compatible in the relevant parameters, $\mathrm{S}$ and $\beta$. These results exhibit a symmetry between assignments, arising probably from the quadrupolar splitting exchange between both assignments. 
The results obtained in the $\mathrm{N}_{t b}$ phase are in agreement with a twisted and bent collective molecular arrangement in chiral domains as the detected molecular ordering reveals a tilted and twisted local molecular packing of the dimer mesogenic groups.

\section{AUTHOR INFORMATION}

Corresponding Authors

*E-mail:carlos.cruz@tecnico.ulisboa.pt

*E-mail:joao.figueirinhas@tecnico.ulisboa.pt

ORCID

Carlos Cruz:0000-0002-1415-0929

João Figueirinhas:0000-0001-9254-1864

Georg Mehl:0000-0002-2421-4869

Notes

The authors declare no competing financial interest

\section{Acknowledgements}

The authors acknowledge financial support from: - Portuguese Science and Technology Foundation (FCT) through CeFEMA strategic project UID/CTM/04540/2013; - European Union through Grant FP7-PEOPLE-2007-1-1-ITN-215884 (DENDREAMERS); - European Union and the Portuguese Science and Technology Foundation (FCT) through Grant M-ERANET2/0006/2016 (CellColor);

\section{References}

(1) Chen, D.; Nakata, M.; Shao, R.; Tuchband, M. R.; Shuai, M.; Baumeister, U.; Weissflog, W.; Walba, D. M.; Glaser, M. A.; Maclennan, J. E. et al. Twist-bend heliconical 
chiral nematic liquid crystal phase of an achiral rigid bent-core mesogen. Phys. Rev. E: Stat. Phys. Plasmas Fluids Relat. Interdisciplin. Top. 2014, 89, 022506.

(2) Borshch, V.; Kim, Y.-K.; Xiang, J.; Gao, M.; Jákli, A.; Panov, V.; Vij, J.; Imrie, C.; Tamba, M. G.; Mehl, G. H. et al. Nematic twist-bend phase with nanoscale modulation of molecular orientation. Nat. Commun. 2013, 4, 2635.

(3) Dozov, I. On the spontaneous symmetry breaking in the mesophases of achiral bananashaped molecules. EPL 2001, 56, 247-253.

(4) Memmer, R. Liquid crystal phases of achiral banana-shaped molecules: a computer simulation study. Liq. Cryst. 2002, 29, 483-496.

(5) Barnes, P. J.; Douglass, A. G.; Heeks, S. K.; Luckhurst, G. R. An enhanced odd-even effect of liquid crystal dimers Orientational order in the $\alpha, \omega$-bis(4'-cyanobiphenyl-4yl)alkanes. Liq. Cryst. 1993, 13, 603-613.

(6) Cestari, M.; Diez-Berart, S.; Dunmur, D. A.; Ferrarini, A.; de la Fuente, M. R.; Jackson, D. J. B.; Lopez, D. O.; Luckhurst, G. R.; Perez-Jubindo, M. A.; Richardson, R. M. et al. Phase behavior and properties of the liquid-crystal dimer 1",7"-bis(4cyanobiphenyl-4'-yl) heptane: A twist-bend nematic liquid crystal. Phys. Rev. E: Stat. Phys. Plasmas Fluids Relat. Interdisciplin. Top. 2011, 84, 031704.

(7) Robles-Hernández, B.; Sebastián, N.; de la Fuente, M. R.; López, D. O.; Diez-Berart, S.; Salud, J.; Ros, M. B.; Dunmur, D. A.; Luckhurst, G. R.; Timimi, B. A. Twist, tilt, and orientational order at the nematic to twist-bend nematic phase transition of $1^{\prime \prime}, 9^{\prime \prime}$-bis(4cyanobiphenyl-4'-yl) nonane: A dielectric, ${ }^{2} \mathrm{H}$ NMR, and calorimetric study. Phys. Rev. E: Stat. Phys. Plasmas Fluids Relat. Interdisciplin. Top. 2015, 92, 062505.

(8) Panov, V. P.; Nagaraj, M.; Vij, J. K.; Panarin, Y. P.; Kohlmeier, A.; Tamba, M. G.; Lewis, R. A.; Mehl, G. H. Spontaneous Periodic Deformations in Nonchiral Planar- 
Aligned Bimesogens with a Nematic-Nematic Transition and a Negative Elastic Constant. Phys. Rev. Lett. 2010, 105, 167801.

(9) Tripathi, C. S. P.; Losada-Pérez, P.; Glorieux, C.; Kohlmeier, A.; Tamba, M.-G.; Mehl, G. H.; Leys, J. Nematic-nematic phase transition in the liquid crystal dimer CBC9CB and its mixtures with 5CB: A high-resolution adiabatic scanning calorimetric study. Phys. Rev. E: Stat. Phys. Plasmas Fluids Relat. Interdisciplin. Top. 2011, 84, 041707.

(10) Beguin, L.; Emsley, J. W.; Lelli, M.; Lesage, A.; Luckhurst, G. R.; Timimi, B. A.; Zimmermann, H. The Chirality of a Twist-Bend Nematic Phase Identified by NMR Spectroscopy. J. Phys. Chem. B 2012, 116, 7940-7951.

(11) Emsley, J.; Lelli, M.; Lesage, A.; Luckhurst, G. R. A Comparison of the Conformational Distributions of the Achiral Symmetric Liquid Crystal Dimer CB7CB in the Achiral Nematic and Chiral Twist-Bend Nematic Phases. J. Phys. Chem. B 2013, 117, 65476557.

(12) Dong, R. Y.; Kohlmeier, A.; Tamba, M. G.; Mehl, G. H.; Burnell, E. E. Solute NMR study of a bimesogenic liquid crystal with two nematic phases. Chem. Phys. Lett. 2012, 552, 44-48.

(13) Burnell, E. E.; Dong, R. Y.; Kohlmeier, A.; Tamba, M. G.; Welch, C.; Mehl, G. H. NMR Study of a Bimesogenic Liquid Crystal with Two Nematic Phases. Mol. Cryst. Liq. Cryst. 2015, 610, 100-107.

(14) Burnell, E. E.; Ahmed, Z.; Welch, C.; Mehl, G. H.; Dong, R. Y. Deuteron and proton NMR study of D-2, p-dichlorobenzene and 1,3,5-trichlorobenzene in bimesogenic liquid crystals with two nematic phases. Chem. Phys. Lett. 2016, 659, 48-54.

(15) Cifelli, M.; Domenici, V.; Dvinskikh, S.; Luckhurst, G.; Timimi, B. The twist-bend ne- 
matic phase: translational self-diffusion and biaxiality studied by $1 \mathrm{H}$ nuclear magnetic resonance diffusometry. Liq. Cryst. 2017, 44, 204-218.

(16) Adlem, K.; Copic, M.; Luckhurst, G. R.; Mertelj, A.; Parri, O.; Richardson, R. M.; Snow, B. D.; Timimi, B. A.; Tuffin, R. P.; Wilkes, D. Chemically induced twist-bend nematic liquid crystals, liquid crystal dimers, and negative elastic constants. Phys. Rev. E: Stat. Phys. Plasmas Fluids Relat. Interdisciplin. Top. 2013, 88, 022503.

(17) Hoffmann, A.; Vanakaras, A. G.; Kohlmeier, A.; Mehl, G. H.; Photinos, D. J. On the structure of the Nx phase of symmetric dimers: inferences from NMR. Soft Matter 2015, 11, 850-855.

(18) Emsley, J. W.; Lelli, M.; Joy, H.; Tamba, M.-G.; Mehl, G. H. Similarities and differences between molecular order in the nematic and twist-bend nematic phases of a symmetric liquid crystal dimer. Phys. Chem. Chem. Phys. 2016, 18, 9419-9430.

(19) Hori, K.; Iimuro, M.; Nakao, A.; Toriumi, H. Conformational diversity of symmetric dimer mesogens, alpha,omega-bis (4,4 '-cyanobiphenyl)octane, -nonane, alpha,omegabis(4-cyanobiphenyl-4 '-yloxycarbonyl)propane, and -hexane in crystal structures. J. Mol. Struct. 2004, 699, 23-29.

(20) Krzyzewska, K.; Jaroch, T.; Maranda-Niedbala, A.; Pociecha, D.; Gorecka, E.; Ahmed, Z.; Welch, C.; Mehl, G. H.; Pron, A.; Nowakowski, R. Supramolecular organization of liquid-crystal dimers - bis-cyanobiphenyl alkanes on HOPG by scanning tunneling microscopy. Nanoscale 2018, 10, 16201-16210.

(21) Zhu, C. H.; Tuchband, M. R.; Young, A.; Shuai, M.; Scarbrough, A.; Walba, D. M.; Maclennan, J. E.; Wang, C.; Hexemer, A.; Clark, N. A. Resonant Carbon K-Edge Soft X-Ray Scattering from Lattice-Free Heliconical Molecular Ordering: Soft Dilative Elasticity of the Twist-Bend Liquid Crystal Phase. Phys. Rev. Lett. 2016, 116, 147803. 
(22) Stevenson, W. D.; Ahmed, Z.; Zeng, X. B.; Welch, C.; Ungar, G.; Mehl, G. H. Molecular organization in the twist-bend nematic phase by resonant X-ray scattering at the Se K-edge and by SAXS,WAXS and GIXRD. Phys. Chem. Chem. Phys. 2017, 19, 1344913454.

(23) Emsley, J. In Nuclear Magnetic Resonance of Liquid Crystal; Emsley, J., Ed.; Nato ASI Series, 1983; Chapter 15, pp 379-412.

(24) Salili, S. M.; Tamba, M. G.; Sprunt, S. N.; Welch, C.; Mehl, G. H.; Jakli, A.; Gleeson, J. T. Anomalous Increase in Nematic-Isotropic Transition Temperature in Dimer Molecules Induced by a Magnetic Field. Phys. Rev. Lett. 2016, 116, 217801.

(25) Ernst, R. R.; Bodenhausen, G.; Wokaun, A. In Principle of Nuclear Magnetic Resonance in One and Two Dimensions; J.S. Rowlinson, F., Ed.; Oxford Science Publications, 1992; Chapter 2, pp 44-49.

(26) Lounila, J.; Jokisaari, J. Anisotropies in spin-spin coupling constants and chemical shifts as determined from the NMR spectra of molecules oriented in liquid crystal solvents. Prog. Nucl. Magn. Reson. Spectrosc. 1982, 15, 249-290.

(27) Veracini, A. In Nuclear Magnetic Resonance of Liquid Crystal; J.W.Emsley,, Ed.; Nato ASI Series, 1983; Chapter 5, pp 99-118.

(28) Goldstein, H.; Poole, C.; J., S. In Classical Mechanics 3rd edition; Goldstein, H., Poole, C., J., S., Eds.; Adison Wesley, 2000.

(29) Cohen-Tannoudji, C.; Diu, B.; Laloe, F. Quantum mechanics; Wiley, 1977.

(30) Kimmich, R. NMR: Tomography, Diffusometry, Relaxometry; Springer Berlin Heidelberg, 1997.

(31) Press, W. H.; Teukolsky, S. A.; Vetterling, W. T.; Flannery, B. P. Numerical Recipes in $C++$ : The Art of Scientific Computing; Cambridge University Press, 2002. 
(32) Slichter, C. Principles of Magnetic Resonance; Springer Series in Solid-State Sciences; Springer Berlin Heidelberg, 1996.

(33) Ref. 27, p. 114.

(34) Mensio, O.; Zamar, R. C.; Anoardo, E.; Acosta, R. H.; Dong, R. Y. On the role of collective and local molecular fluctuations in the relaxation of proton intrapair dipolar order in nematic 5CB. J. Chem. Phys. 2005, 123, 204911.

(35) Aluculesei, A.; Cachitas, H.; Carvalho, J.; Chavez, F. V.; Figueirinhas, J. L.; Sebastião, P. J.; Cruz, C.; Tamba, M. G.; Kohlmeier, A.; Mehl, G. H. 1H NMR study of molecular order and dynamics in CBC9CB Liquid Crystal. Submitted 2018,

(36) Courtieu, J.; Aroulanda, C.; Lesot, P.; Meddour, A.; Merlet, D. Evolution of the Saupe order parameters of enantiomers from a racemic to a non-racemic liquid crystal solvent: an original light on the absolute configuration determination problem. Liq. Cryst. 2010, 37, 903-912. 


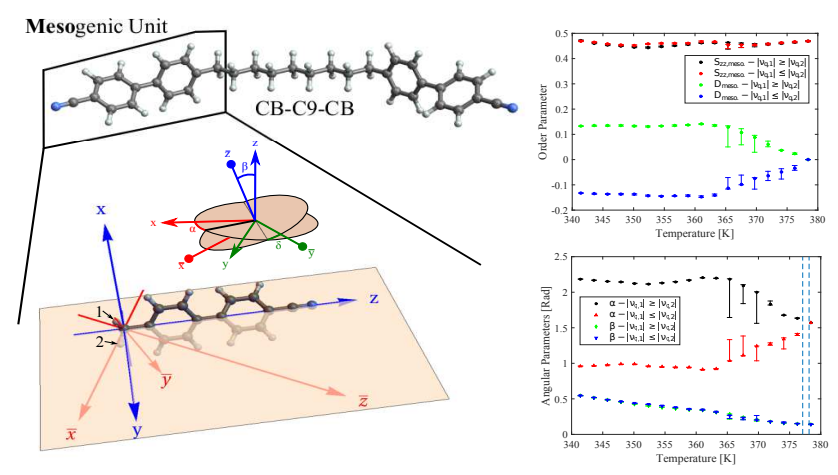

